\title{
Evaluation of a New Point-of-Care HbA1c Assay: The Pixotest
}

\author{
Lau CS ${ }^{1}$, Tong $\mathrm{CH}^{2}$, Goh $\mathrm{R}^{2}$, Aw TC ${ }^{1,3 *}$ \\ ${ }^{1}$ Department of Laboratory Medicine, Changi General Hospital, Singapore. \\ ${ }^{2}$ Faculty of Science, National University of Singapore, Singapore. \\ ${ }^{3}$ Department of Medicine, Yong Loo Lin School of Medicine, National University of Singapore, Singapore. \\ *Corresponding Author: Aw TC, Department of Laboratory Medicine, CHANGI GENERAL HOSPITAL, \\ 2 SIMEI STREET 3, SINGAPORE 529889.
}

\begin{abstract}
:
Introduction: The PixoTest (iXensor) has been introduced for point of care testing (POCT) of glycated hemoglobin (HbAlc), utilizing a colorimetric assay to assess HbAlc on a handheld device with a short turnaround time of around 3 minutes. Such POCT would be very useful in the assessment and diagnosis of diabetes in the outpatient setting. We evaluated the PixoTest's analytical performance for HbAlc against our automated laboratory Cobas (Roche Diagnostics) analyser.
\end{abstract}

Methods: The performance evaluation included assay linearity, analytical precision, Bland-Altman analysis and regression analysis against the Roche Cobas c513 turbidimetric HbAlc assay. Statistical analyses were performed using MedCalc software v18.11.3 (MedCalc, Ostend, Belgium). Our laboratory is a CAP accredited laboratory and both HbAlc assays are NGSP certified.

Results: The PixoTest assays were linear for HbAlc from 4.8-15.0\%. Inter-assay precision (n=20) of Roche controls was satisfactory with a \%CV of of 3.7 and 3.1 respectively. Bland-Altman analysis showed no significant difference between the Cobas and Pixos HbAlc (-0.07\%, $p=0.27)$. Deming Regression analyses showed a close correlation coefficient of $r=0.96$ between the 2 methods.

Conclusion: The performance of HbAlc assay on the PixoTest is good, within the manufacturer's claims, comparable to the Roche assays and fit for operational use.

Keywords: Diabetes, HbAlc, Evaluation, Point-of-care, PixoTest

\section{INTRODUCTION}

Glycated hemoglobin (HbA1c) is recommended by the American Diabetes Association (ADA) for the diagnosis, monitoring, and screening of diabetes/prediabetes [1]. HbA1c correlates with microvascular and macrovascular complications of diabetes [2-6]. The within-subject biological variation of $\mathrm{HbA} 1 \mathrm{c}$ is smaller (3.6\%) when compared to fasting plasma glucose (FPG - 5.7\%) and the oral glucose tolerance test (oGTT $-16.7 \%$ ) [7]. HbA1c assays are internationally harmonized due to the National Glycohemoglobin Standardization Program (NGSP), with all-method precision (coefficient of variation - CV) consistently $<3.0 \%$ over their past 8 surveys [8].

Modern assessments of $\mathrm{HbA1c}$ use either separation techniques (ion exchange chromatography, capillary electrophoresis and affinity chromatography) or chemical methods (immunoassays and enzymatic assays) [9]. HbA1c point of care testing (POCT) is growing in demand due to the convenience and speed of testing allowing same session decision-making in the clinic. The PixoTest (iXensor, Taiwan) is a recently introduced NGSP-certified handheld POCT device for HbA1c with a short turn-around time of 3 minutes. We evaluated the analytical performance of the PixoTest HbA1c against our laboratory automated HbA1c analyser - the Cobas c513 (Roche Diagnostics, Asia-Pacific, Singapore). 


\section{MeTHODS}

The PixoTest Hba1c assay is an immunocolorimetric assay. Ethylenediaminetetraacetic acid (EDTA) whole blood $(5 \mathrm{uL})$ is acquired by capillary action into a proprietary calibrated transfer pipette containing blue-dyed latex microparticles conjugated to specific antibodies directed against the Nterminus of the hemoglobin A0 beta chain. Thereafter, the admixture is added into a buffer solution containing a precise amount of hemolyzing reagent followed by 6-8 agitations to lyse the RBCs. This entire buffer mixture is then loaded onto a test strip coated with immobilized anti-HbA1c antibody. The intensity of resultant blue conjugate on the strip is measured optically and the HbA1c result is available after 3 minutes. The PixoTest has a reported HbA1c measuring range of 4.0-15.0\%. The Cobas c513 is an NGSP-certified immuno-turbidimetric inhibition assay using the Roche Tina-quant reagents [10, 11]. The PixoTest HbA1c assay attained NGSP certification in 2017.

Linearity was studied following the Clinical and Laboratory Standards Institute (CLSI) EP-6 protocol [12]. Patient samples collected in EDTA tubes (Becton-Dickinson, Singapore) with low and high $\mathrm{HbA1c}$ values were mixed to generate samples across a wide range of HbA1c concentrations. Precision was assessed according to the CLSI EP05-A3 protocol [13]. For method comparison, we used the CLSI EP-9 method [14]. Anonymised leftover EDTA whole blood samples that had been previously tested on the Cobas c513 for HbA1c across a broad clinically relevant range $(n=100)$ were recruited and stored at $4^{\circ} \mathrm{C}$. These samples were mixed thoroughly and examined on the PixoTest within 16 hours. As this work was part of our routine evaluation of new diagnostic assays it was exempt from institutional review board approval. Our laboratory is certified by the College of American Pathologists (CAP) and our performance for the Cobas c513 HbA1c assay on the CAP external quality assurance program has been satisfactory. Statistical analyses including Bland-Altman analysis and correlation/regression were performed using MedCalc software v18.11.3 (MedCalc, Ostend, Belgium).

\section{Results}

The PixoTest assay was linear for HbA1c from 4.8-15.0\% against a claimed measuring range of 415\%. Inter-assay precision (CV\%) was $3.7 \%$ at $\mathrm{HbA} 1 \mathrm{c}$ level of $4.8 \%$ and $3.1 \%$ at $\mathrm{HbA1c}$ level of $8.0 \%$ (package insert claimed CV was $3.2 \%$ and $3.5 \%$ for $\mathrm{HbA} 1 \mathrm{c}$ levels of $5.73 \%$ and $8.35 \%$ respectively). Bland-Altman analysis showed no significant difference between the Cobas and PixoTest assays, with a mean difference in HbA1c of $-0.07 \%$ (95\% CI $-0.1909 \%$ to $0.055 \%$ ) (See Figure 1). Deming regression analysis (Figure 2) showed close agreement ( $\mathrm{r}=0.96$; 95\% CI 0.94360.9742) between PixoTest and Roche HbA1c (stated $r=0.99$ against the Tosoh G7 analyzer).

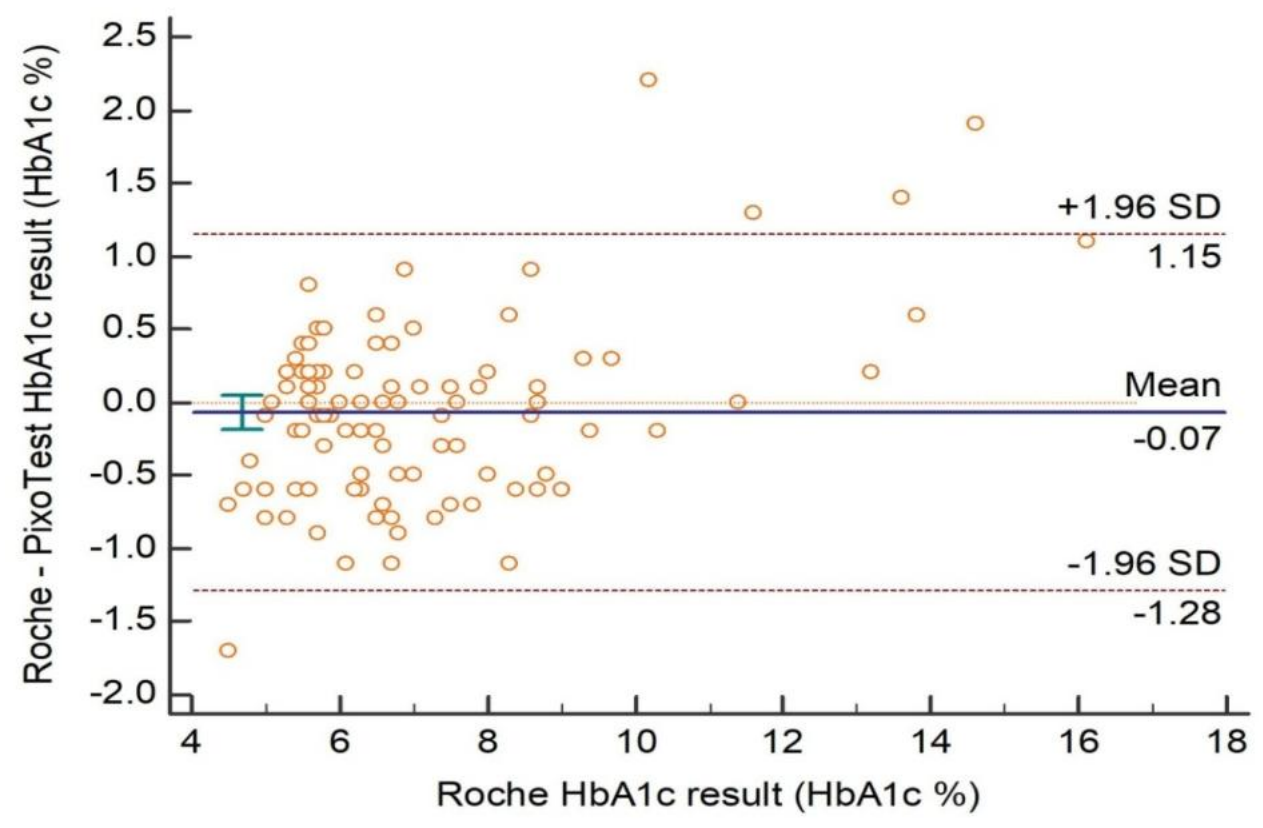

Figure1. Bland-Altman analysis of PixoTest versus Cobas c513 HbAlc results 


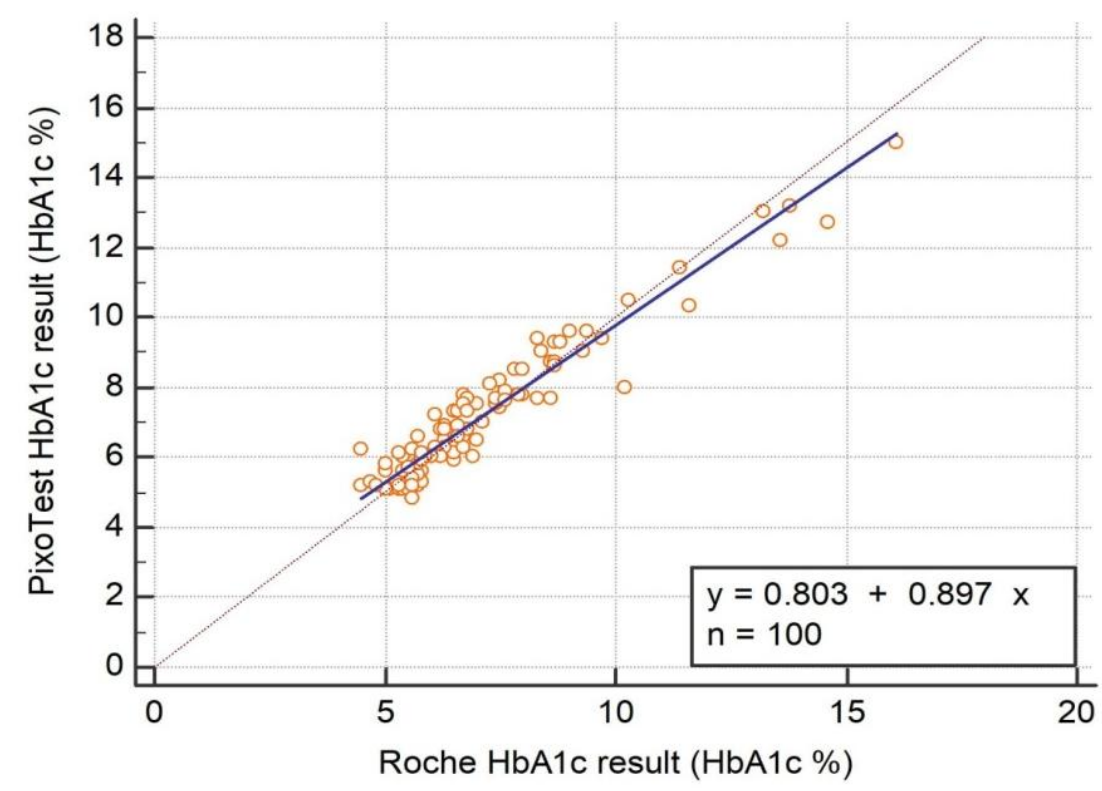

Figure2. Deming regression analysis of PixoTest versus Cobas c513 HbAlc

\section{DISCUSSION}

Our evaluation of the PixoTest HbA1c assay confirms that its performance is good, within the manufacturer's claims and is comparable to the Cobas c513. The PixoTest assay precision was quite acceptable $-3.1 \%$ for the high HbA1c control $(8.0 \%)$ and $3.7 \%$ for the low control $(4.8 \%)$. To simulate real-world conditions, precision testing was performed by 4 operators on 4 different instruments. The PixoTest is fit for operational use. However, several other NGSP-certified HbA1c POCT devices are available [15-17] (see Table 1). They all have good precision (CVs <4\%) in the clinically relevant range. The newer devices [17] and the PixoTest have improved assay time $(<4$ $\min )$.

Table 1: Comparison of HbA1c POCT devices

\begin{tabular}{|c|c|c|c|c|}
\hline \multirow{2}{*}{ Name } & \multirow{2}{*}{ Assay type } & \multirow{2}{*}{$\begin{array}{l}\text { Assay time } \\
\text { (min) }\end{array}$} & \multicolumn{2}{|c|}{ HbA1c Precision } \\
\hline & & & Concentration (\%) & CV (\%) \\
\hline \multirow{2}{*}{$\begin{array}{l}\text { DCA Vantage } \\
\text { (Siemens) }\end{array}$} & \multirow{2}{*}{$\begin{array}{l}\text { Latex agglutination } \\
\text { inhibition immunoassay }\end{array}$} & \multirow{2}{*}{6} & $5.1 / 11.2^{(125)}$ & $1.8 / 3.7$ \\
\hline & & & $5.3 / 6.1 / 8.1^{(16)}$ & $2.3 / 2.5 / 2.7$ \\
\hline \multirow{2}{*}{$\begin{array}{c}\text { Afinion } \\
\text { (Axis-Shield) }\end{array}$} & \multirow{2}{*}{ Affinity separation } & \multirow{2}{*}{10} & $4.7 / 6.3 / 8.2^{(15)}$ & $2.4 / 1.4 / 1.8$ \\
\hline & & & $5.3 / 6.1 / 8.1^{(16)}$ & $2.8 / 3.1 / 2.4$ \\
\hline $\begin{array}{l}\text { Afinion2 } \\
\text { (Abbott) }\end{array}$ & Affinity separation & 3 & $6.2 / 9.0^{(17)}$ & $1.2 / 0.9$ \\
\hline $\begin{array}{c}\text { Quo-Lab } \\
\text { (EKF Diagnostics) }\end{array}$ & $\begin{array}{l}\text { Affinity separation and } \\
\text { fluorescence quenching }\end{array}$ & 4 & $6.4 / 8.6^{(17)}$ & $1.6 / 1.8$ \\
\hline $\begin{array}{l}\text { PixoTest } \\
\text { (iXensor) }\end{array}$ & Immunocolorimetric & 3 & $4.8 / 8.0$ & $3.7 / 3.1$ \\
\hline
\end{tabular}

This report is novel in that the PixoTest HbA1c is a new assay that has not been reported before. It adds to the body of literature and provides additional information for consideration when selecting a POCT device for HbA1c [16]. The utility of HbA1c is growing. Studies [18] show that POCT HbA1c is superior to regular diabetes screening, diagnosing $63 \%$ of the population to be dysglycemic (prediabetic or diabetic) compared to $41 \%$. HbA1c also has a greater concordance with pre-diabetes and diabetes [19] than oGTT. Besides, HbA1c has a stronger association with cardiovascular disease than oGTT or fasting plasma glucose (FPG) [20]. An Australian study performed on an Aboriginal 
community [21] at high risk of developing diabetes showed good concordance between a POCT HbA1c (Siemens DCA 2000+) and the Roche Cobas HbA1c.

A slight limitation of this PixoTest device is the need for adequate mixing of the test sample with the hemolyzing reagent and the completeness of transferring the entire reaction contents onto the measuring cup of the test strip. Moreover, we did not test the effect of $\mathrm{Hb}$ variants on this system. While hemoglobin $(\mathrm{Hb})$ variants are the bane of $\mathrm{HbAlc}$ measurements, the effect of $\mathrm{Hb}$ variants on modern HbA1c immunoassays is becoming less of a concern. Except for homozygous hemoglobinopathy, heterozygous variant states have minimal effects on HbA1c results. A recent study [22] comparing 100 normoglycemic beta-thalassemia carriers and 100 controls without diabetes showed that the mean HbA1c concentration on the Cobas Integra 800 analyzer was almost identical in both groups $-5.23 \%$ versus $5.22 \%$. Besides, homozygous hemoglobinopathies are extremely rare in our practice. In one national referral center near our hospital, $\mathrm{Hb}$ variants were encountered in $2.3 \%$ (129/5628) of the HbA1c tests done over a 5 month period [23]; of these only $5(0.09 \%)$ were homozygous hemoglobinopathies.

\section{CONCLUSION}

The PixoTest HbA1c POCT device has excellent correlation with the Cobas c513 auto-analyzer results with no significant differences. As $\mathrm{HbA1c}$ is recommended for the screening, diagnosis and monitoring of diabetes, rapid HbA1c testing using accurate POCT devices is desirable. With a rapid assay time of 3 minutes, PixoTest can aid the clinician in quick decision-making.

\section{Acknowledgments}

We thank iXensor (Taiwan) for generously providing the PixoTest HbAlc analyser and teststrips for the study. iXensor was not involved in the study design or the writing of this manuscript.

\section{Author Contributions}

All authors have made substantial contributions (design of study, data acquisition, drafting article and approval of final version for submission).

\section{REFERENCES}

[1] American Diabetes Association. 2. Classification and Diagnosis of Diabetes: Standards of Medical Care in Diabetes - 2019. Diabetes Care 2019; 42: 13-28.

[2] The Diabetes Control and Complications Trial Research Group. The effect of intensive treatment of diabetes on the development and progression of long-term complications in insulin-dependent diabetes mellitus. N Engl J Med 1993; 329: 977-986.

[3] The Diabetes Control and Complications Trial Research Group. The relationship of glycemic exposure (HbA1c) to the risk of development and progression of retinopathy in the diabetes control and complications trial. Diabetes 1995; 44: 968-983.

[4] UK Prospective Diabetes Study (UKPDS) Group. Intensive blood-glucose control with sulphonylureas or insulin compared with conventional treatment and risk of complications in patients with type 2 diabetes (UKPDS 33). Lancet 1998; 352: 837-853.

[5] UK Prospective Diabetes Study (UKPDS) Group. Effect of intensive blood-glucose control with metformin on complications in overweight patients with type 2 diabetes (UKPDS 34). Lancet 1998; 352: 854-865.

[6] Colagiuri S, Lee CM, Wong TY, Balkau B, Shaw JE, Borch-Johnsen K, et al. Glycemic thresholds for diabetes-specific retinopathy: implications for diagnostic criteria for diabetes. Diabetes Care 2011;34: 145-150.

[7] Florkowski C. HbA1c as a diagnostic test for Diabetes Mellitus - reviewing the evidence. Clin Biochem Rev 2013; 34: 75-83.

[8] Little RR, Rohlfing C, Sacks DB. The National Glycohemoglobin Standardization Program: over 20 Years ofImproving Hemoglobin A1c measurement. Clin Chem 2019; 65: 839-848.

[9] Weykamp C. HbA1c: areview of analytical and clinical aspects. Ann Lab Med 2018; 33: 393-400.

[10] Lenters-Westra E, English E. Evaluating new HbA1c methods for adoption by the IFCC and NGSP reference networks using international quality targets. Clin Chem Lab Med 2017; 55: 1426-1434. 
[11] Jaisson S, Leroy N, Soulard M, Desmons A, Guillard E, Gillery P. Evaluation of the analytical performances of the Cobas c513 analyser for HbA1c assay. Biochem Med (Zagreb) 2018; 28: 030708, doi: 10.11613/BM.2018.030708.

[12] Clinical and Laboratory Standard Institute (CLSI). Evaluation of the Linearity of Quantitative Measurement Procedures: A Statistical Approach; Approved Guideline, CLSI Document EP06-A. Wayne PA: CLSI 2003.

[13] Clinical and Laboratory Standard Institute (CLSI). Evaluation of Precision of Quantitative Measurement Procedures; Approved Guideline-Third Edition, CLSI document EP05-A3. PA Wayne PA: CLSI 2014.

[14] Clinical and Laboratory Standard Institute (CLSI). Measurement Procedure Comparison and Bias Estimation Using Patient Samples; Approved Guideline-Third Edition, CLSI document EP09-A3. Wayne PA: CLSI 2013.

[15] Lenters-Westra E, Slingerland RJ. Six of eight hemoglobin A1c point-of-care instruments do not meet the general accepted analytical performance criteria. Clin Chem 2010; 56: 44-52.

[16] Whitley HP, Yong EV, Rasinen C. Selecting an A1C point-of-care instrument. Diabetes Spectr 2015; 28 : 201-208.

[17] Lenters-Westra E, English E. Evaluation of four HbA1c point-of-care devices using international quality targets: are they fit for the purpose. J Diabetes Sci Technol 2018; 12: 762-770.

[18] Whitley HP, Hanson C, Parton JM. Systematic diabetes screening using point-of-care HbA1c testing facilitates identification of Prediabetes. Ann Fam Med 2017; 15: 162-164.

[19] Chatzianagnostou K, Vigna L, Di Piazza S, Tirelli AS, Napolitano F, Tomaino L, et al. Low concordance between HbA1c and OGTT to diagnose prediabetes and diabetes in overweight or obesity. Clin Endocrinol (Oxf) 2019; 91: 411-416.

[20] Metcalf PA, Kyle C, Kenealy T, Jackson RT. HbA1c in relation to incident diabetes and diabetes-related complications in non-diabetic adults at baseline. J Diabetes Complications 2017; 31: 814-823.

[21] Marley JV, Oh MS, Hadgraft N, Singleton S, Issacs K, Atkinson D. Cross-sectional comparison of pointof-care with laboratory $\mathrm{HbA1c}$ in detecting diabetes in real-world remote Aboriginal settings. BMJ Open 2015; 5: e006277. doi: 10.1136/bmjopen-2014-006277.

[22] Tsilingiris D, Makrilakis K, Voskaridou E, Pagkrati S, Dalamaga M, Liatis S. Effect of heterozygous beta thalassemia on HbA1c levels in individuals without diabetes mellitus: a cross sectional study. Clin Chim Acta 2019; 494: 132-137.

[23] Saw S, Loh TP, Yin C, Sethi SK. Identification of hemoglobin variants in samples received for glycated hemoglobin testing. Clin Chim Acta 2013; 415: 173-175.

ABbREVIATIONS:

ADA - American Diabetes Association

CAP - College of American Pathologists

CLSI - Clinical and Laboratory Standards Institute

$\mathrm{CV}$ - coefficient of variation

EDTA - Ethylenediaminetetraacetic acid

FPG - Fasting plasma glucose

$\mathrm{Hb}$ - Hemoglobin

HbA1c - Glycated hemoglobin

HPLC - high performance liquid chromatography

NGSP - National Glycohemoglobin Standardization Program

oGTT - oral glucose tolerance test

POCT - Point-of-care testing

Citation: Aw TC, et.al., (2019), "Evaluation of a New Point-of-Care HbAlc Assay: The Pixotest". International Journal of Clinical Chemistry and Laboratory Medicine (IJCCLM), 5(4), pp.1-5, DOI: http://dx.doi.org/10.20431/2455-7153.0504001.

Copyright: (C) 2019 Authors. This is an open-access article distributed under the terms of the Creative Commons Attribution License, which permits unrestricted use, distribution, and reproduction in any medium, provided the original author and source are credited. 